\title{
Generalization of Child-Langmuir Law for Non-Zero Injection Velocities in a Planar Diode
}

\author{
R. R. Puri, Debabrata Biswas and Raghwendra Kumar \\ Theoretical Physics Division \\ Bhabha Atomic Research Centre \\ Mumbai 400 085, INDIA
}

(October 29, 2018)

\begin{abstract}
The Child-Langmuir law relates the voltage applied across a planar diode to the saturation value $J_{\mathrm{CL}}$ of current density that can be transmitted through it in case the injection velocity of electrons into the diode is zero. The Child-Langmuir current density $J_{\mathrm{CL}}$ is, at the same time, (i) the maximum current density that can be transmitted through a planar diode, (ii) the current density below which the flow is steady and unidirectional in the long time limit and (iii) the average transmitted current density for any value of injected current density above $J_{\mathrm{CL}}$. Existing generalizations of Child-Langmuir law to non-zero velocities of injection are based on the characteristics (i) and (ii) of $J_{\mathrm{CL}}$. This paper generalizes the law to non-zero velocities of injection based on the characteristic (iii) by deriving an analytical expression for the saturation value of current density. The analytical expression for the saturation current density is found to be well supported by numerical computations. A reason behind preferring the saturation property of the Child-Langmuir current density as the basis for its generalization is the importance of that property in numerical simulations of high current diode devices.
\end{abstract}

\section{INTRODUCTION}

The celebrated Child-Langmuir law is widely used in many areas of physics where charged particle transport across a potential difference is involved [1]. It gives the maximum current density $J_{\mathrm{CL}}$ that can be transported from an infinite planar cathode at zero potential to an infinite planar anode parallel to the cathode at a distance $L$ and potential $V$ when the initial velocity $v_{0}$ of electrons at the cathode is zero. This maximum value is a result of the space charge effect and, in the non-relativistic limit, is given by

$$
J_{\mathrm{CL}}=\frac{V^{3 / 2}}{L^{2}} \frac{1}{9 \pi}\left(\frac{2 e}{m}\right)^{1 / 2} .
$$

Here, $e$ is the magnitude of the charge on an electron and $m$ is its rest mass. For convenience, the terms "current" and "current density" are used interchangeably. They refer to the current density denoted by $J$.

There have been several attempts to generalize the law (1) to other situations such as to higher dimensions or to non-zero velocities of injection [2]- [6]. The characteristics of $J_{\mathrm{CL}}$ on which such generalizations are based are one or the other of the following: (i) $J_{\mathrm{CL}}$ is the maximum current that can be transmitted across one-dimensional planar diode with zero velocity of injection, and (ii) the flow of current in the long time limit is steady and unidirectional if the injected current is less than $J_{\mathrm{CL}}$ but oscillatory in time above it. The generalization based on characteristic (i) to non-zero injection velocity, $v_{0}$, of electrons in to a planar diode shows that the space charge limiting current

$$
J_{\mathrm{SCL}}=J_{\mathrm{CL}}\left[\left(\frac{m v_{0}^{2}}{2 e V}\right)^{1 / 2}+\left(1+\frac{m v_{0}^{2}}{2 e V}\right)^{1 / 2}\right]^{3}
$$

is the maximum current that can be transmitted across the diode and is identified as the generalized Child-
Langmuir current [5]. The generalization based on characteristic (ii), on the other hand, shows that the current in the diode is unidirectional till it attains the value $J_{\mathrm{BF}}$ and starts reflecting thereafter with

$$
J_{\mathrm{BF}}=J_{\mathrm{CL}}\left[\left(\frac{m v_{0}^{2}}{2 e V}\right)^{3 / 4}+\left(1+\frac{m v_{0}^{2}}{2 e V}\right)^{3 / 4}\right]^{2}
$$

This current, $J_{B F}$, marking the bifurcation of the state from completely to partially transmitting is proposed in Ref. [6] as a generalized Child-Langmuir current.

A third characteristic that is commonly ignored in such attempts is the fact that $J_{\mathrm{CL}}$ is the saturation current that is transmitted for zero velocity of injection for any injected current higher than $J_{\mathrm{CL}}$. It is the existence of saturation current in a planar diode that we wish to explore in this communication for non-zero velocities of injection and call the relation between such a saturation current, the injection velocity, the diode spacing and voltage as the generalized Child-Langmuir law.

The reason for emphasising the saturation property is that it helps in simulations involving high current devices. For, the existence of a saturation current implies that one need not bother about the material dependent cathode characteristics (the Richardson-Dushman law in case of thermionic emission or the Fowler-Nordheim law for field emission) so long as the current emitted from the cathode surface is more than what gives rise to the saturation value of the transmitted current. This condition simplifies considerably the task of numerical simulation.

Though the injection velocity of electrons in many devices can be taken as zero, in several others non-zero injection velocities do arise (see [1] and references therein). The magnitude of the velocity can be related to physically measurable quantities such as the temperature in case of thermionic emission or the frequency of the incident field in case of photoemission. Since, as in the case of $v_{0}=0$, existence of a saturation current for $v_{0} \neq 0$ 
may enable one to ignore the cathode characteristics for appropriate injected currents, it is important to know for what values of injected current and to what value, if any, does the transmitted current saturate if $v_{0} \neq 0$. The main result of this paper is that as the current injected in to a planar diode is increased beyond its space charge limiting value, the current transmitted through it saturates asymptotically to the value

$$
J_{\mathrm{GCL}}=J_{\mathrm{CL}}\left(1+\frac{m v_{0}^{2}}{2 e V}\right)^{3 / 2},
$$

which we call the generalized Child-Langmuir current. The equation above is the desired generalization to $v_{0} \neq 0$ of the Child-Langmuir relation (1) for $v_{0}=0$. Note that $J_{\mathrm{GCL}} \leq J_{\mathrm{BF}} \leq J_{\mathrm{SCL}}$ and that the equalities hold when $v_{0}=0$.

The relation (4) is derived in the framework of a phenomenological theory, called the classical theory. The validity of that theory is assessed by comparison with numerical solution of the equations.

The paper is organised as follows. In Sec.2 we recall the main results of the steady state theory leading to the expression for the space charge limiting current. Some details of derivation of those results are outlined in the Appendix A. The classical theory for currents exceeding the space charge limiting current is presented in Sec.3. The results of numerical computation are presented in Sec. 4.

\section{SPACE CHARGE LIMITING CURRENT}

Consider a diode consisting of two infinite parallel plates placed at $z=0$ and $z=L$ where $z$ is the direction orthogonal to the plates. Let an electronic fluid of uniform and same cross-section as the plates move along the z-direction. Its motion in the Eulerian formalism is governed by the equations [7]

$$
\begin{aligned}
& \frac{\partial^{2} \phi}{\partial z^{2}}=-\frac{\partial E}{\partial z}=-4 \pi \rho(z, t) \\
& \frac{\partial \rho}{\partial t}+\frac{\partial J}{\partial z}=0 \\
& \frac{\mathrm{d} p}{\mathrm{~d} t}=-e E(z, t)
\end{aligned}
$$

where $\rho(z, t)$ and $J(z, t)$ are respectively the charge and current densities of the electronic fluid, $p(z, t)$ is the momentum of the electron whereas $\phi(z, t)$ and $E(z, t)$ are the potential and the electric field at the position $z$ at time $t$.

We consider the case when a stream of electrons of constant charge density $\rho_{0}$ enters the diode at $z=0$ with each electron in the stream entering with the same velocity $v_{0}$ along the $z$-direction so that

$$
\rho(0, t)=\rho_{0}, \quad J(0, t) \equiv \rho(0, t) v_{0}=J_{0} .
$$

Furthermore, the plates at $z=0$ and $z=L$ are assumed to be maintained at fixed potentials given by

$$
\phi(0, t)=0, \quad \phi(L, t)=V .
$$

Because of the defining relation between the field and the potential, the condition (7) may be rewritten as

$$
\int_{0}^{L} E(z, t) \mathrm{d} z=V .
$$

A complete description of the motion of the electronic fluid is provided by solving equations (5) under the boundary condition (7) or (8).

A state of interest, called a steady state, is the one that the system reaches asymptotically in time i.e. as $t \rightarrow \infty$. The evaluation of space charge limiting current requires solving the steady state form of the equations obtained by equating to zero the partial derivatives with respect to time in the Eulerian formalism. It is evident from (5) that the current density in this case is not only independent of time but is also the same at every plane. If there is no reflection of electrons from anywhere in the diode then the current density in the diode is everywhere the same as is pumped in at its entrance so that, in the steady state, $J(z, t) \equiv \rho(z, t) v(z, t)=J_{0}$. The first of the equations in (5) then assumes the form

$$
\frac{\mathrm{d}^{2} \phi}{\mathrm{d} z^{2}}=-\frac{4 \pi J_{0}}{v} .
$$

The relation between $\phi$ and $v$ is provided by the steady state form of the law of conservation of energy,

$$
\frac{1}{2} m v^{2}-e \phi=\frac{1}{2} m v_{0}^{2} \equiv \varepsilon
$$

where we have introduced the parameter $\varepsilon$ to denote the electronic energy and assumed that $\varepsilon$ is small enough for the motion to be considered non-relativistic. On combining (9) and (10) we arrive at the equation

$$
\frac{\mathrm{d}^{2} \bar{\phi}}{\mathrm{d} \bar{z}^{2}}=\frac{4 \alpha}{9 \sqrt{\bar{\phi}}}
$$

where the scaled current $\alpha$ and the scaled potential $\bar{\phi}$ are defined by

$$
\begin{aligned}
& \alpha=9 \pi e\left|J_{0}\right| L^{2} \varepsilon^{-3 / 2} \sqrt{\frac{m}{2}}, \\
& \bar{\phi}=1+\frac{e \phi}{\varepsilon} .
\end{aligned}
$$

In terms of the scaled potential, the boundary condition (7) assumes the form

$$
\bar{\phi}(z=0, t)=1, \quad \bar{\phi}(z=L, t)=\bar{V},
$$

where

$$
\bar{V}=1+\frac{e V}{\varepsilon} .
$$

Though the steady state may be determined by solving (11) along with the boundary conditions (14), it turns 
out to be more convenient to derive it instead in the Lagrangian formalism. We will, however, return to Eq.(11) in the next section which concerns the situation involving returning currents.

Alternative to the Eulerian formalism which characterizes the motion in terms of hydrodynamical variables like charge and current densities as function of space and time, is the Lagrangian formalism which is based on determining the trajectories of the electrons. An electronic trajectory in the one-dimensional motion under consideration is identified by the time $t_{0}$ at which it enters the diode. In the non-relativistic limit $(p=m v)$, the trajectory of an electron entering the diode at $t=t_{0}$ is determined by the solution of the Llewellyn's equation

$$
\frac{\partial^{3} z\left(t, t_{0}\right)}{\partial t^{3}}=-\frac{e}{m}\left[\frac{\partial E_{0}(t)}{\partial t}+4 \pi J_{0}(t)\right],
$$

where $E_{0}(t) \equiv E(0, t)$ and $J_{0}(t) \equiv J(0, t)$ are the electric field and the current density at the entrance $(z=0)$ of the diode. The steady state solution of this equation has been derived in Ref. [5]. However, for the sake of completeness and clarity, and in order to fill the gaps in the available derivations, we present in Appendix A the solution of (16) for the steady state corresponding to unidirectional flow of current in terms of the following dimensionless variables

$$
\begin{aligned}
& \bar{z}=\frac{z}{L}, \quad \bar{t}=\frac{t}{L} \sqrt{\frac{2 \varepsilon}{m}}, \quad \bar{v}=\sqrt{\frac{m}{2 \varepsilon}} v=\frac{v}{v_{0}}, \\
& \bar{E}=\frac{e L E}{\varepsilon}=-\frac{\mathrm{d} \bar{\phi}}{\mathrm{d} \bar{z}} .
\end{aligned}
$$

The injected current $J_{0}$ in the steady state is transmitted completely to the anode where the electrons arrive with velocity $v_{1}$ which, by virtue of the energy conservation relation (10), is given by

$$
\bar{v}_{1}=\sqrt{\bar{V}} .
$$

The main results of the steady state solution are:

1. The steady state is determined by solving the cubic (A9) for the transit time $\bar{T}(1)$ of an electron across the diode. The flow of current is unidirectional and independent of time if that cubic admits physically acceptable solution.

2. The cubic admits two positive roots, $\bar{T}_{2}(1)$ and $\bar{T}_{3}(1)$, given in $(A 14)$, if the value of the injected current $\alpha \leq \alpha_{\mathrm{SCL}}$ where

$$
\alpha_{\mathrm{SCL}}=(1+\sqrt{\bar{V}})^{3}
$$

is the space charge limiting current. By invoking (12), it may be verified that the expression for the injected current $J_{0}$ corresponding to $\alpha$ given above is the same as in (2) and that if $v_{0}=0$ then

$$
J_{\mathrm{SCL}}=J_{\mathrm{CL}}, \quad v_{0}=0,
$$

where $J_{\mathrm{CL}}$ is given by (1). In other words, for zero velocity of injection, the space charge limiting current is the same as the Child-Langmuir current.
3. For $0 \leq \alpha \leq \alpha_{\mathrm{DR}}$, the root $\bar{T}_{3}(1)$ corresponds to negative velocities in certain region of the diode and hence is unacceptable in the said range of the injected current. However, both the roots, $\bar{T}_{2}(1)$ and $\bar{T}_{3}(1)$ are acceptable if $\alpha_{\mathrm{DR}} \leq \alpha \leq \alpha_{\mathrm{SCL}}$ where

$$
\alpha_{\mathrm{DR}}=\left(1+\bar{V}^{3 / 4}\right)^{2} .
$$

The suffix DR on $\alpha$ stands for "double root": it is the value of the current above which steady state equation has two admissible roots. The linear stability analysis shows that, in the range mentioned above, $\bar{T}_{3}(1)$ leads to unstable steady state. Hence, $\bar{T}_{2}(1)$ defines the acceptable stable steady state for all $\alpha \leq \alpha_{\mathrm{SCL}}$.

4. The flow of current ceases to be unidirectional and time-independent if the injected current exceeds $\alpha_{\mathrm{SCL}}$.

The above-mentioned characteristics of the steady state solution are exhibited in Fig. 1 by the plot of the electric field $\bar{E}_{0}$ at the cathode evaluated using (A16) for $\bar{V}=1.2$. The point $A$ in the figure corresponds to the space charge limiting current $\alpha_{\mathrm{SCL}}$ whereas the point $B$ represents $\alpha_{\mathrm{DR}}$. The branches $\mathrm{OA}$ and $\mathrm{AB}$ in that figure correspond, respectively, to $\bar{T}_{2}(1)$ and $\bar{T}_{3}(1)$ given by (A14). The branch AB represents unstable steady state. The uppermost branch of the curve for $\alpha>\alpha_{\mathrm{DR}}$ is a result of the classical theory outlined in the next section. The value of $\bar{E}_{0}$ at $\alpha_{\mathrm{DR}}$ where the unstable steady state corresponding to $\bar{T}_{3}(1)$ merges with the classical theory, found using (A16), is given by

$$
\bar{E}_{0 \mathrm{DR}}=\frac{4}{3}\left(1+\bar{V}^{3 / 4}\right) .
$$

We will see that, as it should be, this value is the same as that obtained by the classical theory.

By transforming back to the unnormalized variables, the results above may be applied to the case of $v_{0}=0$. It may be verified that the expression for space charge limiting current density $J_{0}$, found using (19) in this case is the well-known Child-Langmuir current $J_{\mathrm{CL}}$ given in (1) and that, correspondingly, $E_{0}=0$. We thus see that, contrary to the standard treatments of the case of $v_{0}=0$, $E_{0}=0$ is not an imposed condition but is a natural consequence of the governing equations of motion. This fact has also been emphasised in [5].

Since the space charge limiting current $\alpha_{\mathrm{SCL}}$ is the maximum current that can be transmitted across the diode, it is proposed in [5] that $\alpha_{\mathrm{SCL}}$ may be identified as the generalized Child-Langmuir current and (19) as the generalized Child-Langmuir current-voltage relation. However, as we have argued in the Introduction, an important characteristic of Child-Langmuir current is that it is the current transmitted across the diode for any injected current above $\alpha_{\mathrm{SCL}}$. We, therefore, need to examine the behaviour of the transmitted current for $\alpha>\alpha_{\mathrm{SCL}}$.

As shown in the Appendix A, the system does not admit a steady state corresponding to unidirectional flow of 
current if $\alpha>\alpha_{\mathrm{SCL}}$. Hence, the behaviour of the system for $\alpha>\alpha_{\mathrm{SCL}}$ is determined by solving time-dependent equations. Since solving time-dependent equations analytically is a formidable task, those equations are solved numerically. However, a qualitative understanding of the phenomenon is achieved by a simple model which assumes that, though for $\alpha>\alpha_{\text {SCL }}$ the current may not be unidirectional, it nevertheless attains a steady state with current flowing in two directions in some region in the diode. This theory, called the classical theory, is outlined in the next section. It is along the lines of the theory in Ref. [7] for the case of grounded plates.

\section{ASYMPTOTICALLY SATURATING CURRENT}

The analysis outlined in the Appendix A holds good for $\alpha \leq \alpha_{\mathrm{SCL}}$ in which case the injected and the transmitted currents are equal. The classical theory assumes that, beyond $\alpha_{\mathrm{SCL}}$, the minima of the potential within the diode assumes a value which is such that the velocity of an electron there reduces to zero. The equations (10) and (13) show that $v=0$ is attained when $\bar{\phi}=0$. Hence, the boundary condition at the position $\bar{z}=\bar{z}_{m}$ of the potential minima is

$$
\bar{\phi}=\frac{\mathrm{d} \bar{\phi}}{\mathrm{d} \bar{z}}=0, \quad\left(\bar{z}=\bar{z}_{m}\right) .
$$

After the electrons come to rest at $\bar{z}=\bar{z}_{m}$, it is assumed that a fraction $f$ of them is transmitted while the remaining fraction $1-f$ is reflected back towards the cathode. Thus, in the region $0 \leq \bar{z} \leq \bar{z}_{m}$, there are two currents: the injected current $\alpha$ moving away from the cathode and $(1-f) \alpha$ moving towards it. In the region $\bar{z}_{m} \leq \bar{z} \leq 1$, there is unidirectional flow of current of magnitude $f \alpha$ towards the anode. Note that the net current at every plane in the two regions is $f \alpha$. Since the charge densities at a point due to different currents at it add, the Poisson's equation (11) in the two regions assumes the forms

$$
\begin{aligned}
\frac{\mathrm{d}^{2} \bar{\phi}_{L}}{\mathrm{~d} \bar{z}^{2}}=\frac{4 \alpha(2-f)}{9 \sqrt{\bar{\phi}_{L}}}, & 0 \leq \bar{z} \leq \bar{z}_{m}, \\
\frac{\mathrm{d}^{2} \bar{\phi}_{R}}{\mathrm{~d} \bar{z}^{2}}=\frac{4 \alpha f}{9 \sqrt{\bar{\phi}_{R}}}, & \bar{z}_{m} \leq \bar{z} \leq 1 .
\end{aligned}
$$

Both these equations are of the form $\mathrm{d}^{2} \phi / \mathrm{d} z^{2}=C / \phi^{1 / 2}$ and can be recast as

$$
\frac{\mathrm{d}}{\mathrm{d} z}\left(\frac{\mathrm{d} \bar{\phi}}{\mathrm{d} \bar{z}}\right)^{2}=4 C \frac{\mathrm{d} \sqrt{\bar{\phi}}}{\mathrm{d} \bar{z}} .
$$

The solution of this equation, satisfying the boundary condition (23) reads,

$$
\frac{\mathrm{d} \bar{\phi}}{\mathrm{d} \bar{z}}= \pm 2 \sqrt{C} \bar{\phi}^{1 / 4} .
$$

Now, recall that in a one-dimensional electrostatic situation involving charges of only one sign, there can be only one extrema. In the problem at hand, the minima at $\bar{z}=\bar{z}_{m}$, therefore, is the only one admissible so that the electrons are decelerated while moving from the cathode to the position of minima at $\bar{z}=\bar{z}_{m}$ and are accelerated thereafter. Hence, the minus sign in the solution above holds in the region $0 \leq \bar{z}<\bar{z}_{m}$ whereas the plus sign is to be retained in the region $\bar{z}_{m}<\bar{z}<1$. On integrating (27) in the two regions with appropriate values of $C$ along with the boundary conditions at $\bar{z}=0,1$ given in (14), it follows that

$$
\begin{array}{ll}
\bar{\phi}_{L}^{3 / 4}=1-\bar{z} \sqrt{\alpha(2-f)}, & 0 \leq \bar{z} \leq \bar{z}_{m}, \\
\bar{\phi}_{R}^{3 / 4}=(\bar{z}-1) \sqrt{\alpha f}+\bar{V}^{3 / 4}, & \bar{z}_{m} \leq \bar{z} \leq 1 .
\end{array}
$$

The electric field at the cathode, obtained by substituting (28) corresponding to $\bar{z}=0$ in (27) reads

$$
\bar{E}_{0}=\frac{4 \sqrt{\alpha(2-f)}}{3} .
$$

The unknowns $\bar{z}_{m}$ and $f$ in the expressions above are determined by demanding that $\bar{\phi}$ in the two regions should match at $\bar{z}=\bar{z}_{m}$. To that end, note that, by virtue of (23), the expressions (28) and (29), evaluated at $\bar{z}=\bar{z}_{m}$, lead to the relations

$$
\begin{aligned}
& \bar{z}_{m}=\frac{1}{\sqrt{\alpha(2-f)}}, \\
& \bar{z}_{m}=1-\frac{\bar{V}^{3 / 4}}{\sqrt{\alpha f}} .
\end{aligned}
$$

These equations determine two unknowns, the position $\bar{z}_{m}$ of the plane of reflection and the fraction $f$ of the injected current that is transmitted. On eliminating $\bar{z}_{m}$ between these equations, the expression determining $f$ reads

$$
\sqrt{\alpha}=\frac{1}{\sqrt{2-f}}+\frac{\bar{V}^{3 / 4}}{\sqrt{f}} .
$$

It is straightforward to verify that $\mathrm{d} f / \mathrm{d} \alpha<0$. Hence, $f$ is a decreasing function of $\alpha$. Now, (33) shows that the value $f=1$ is attained when $\alpha=\alpha_{\mathrm{BF}}$ where

$$
\alpha_{\mathrm{BF}}=\left(1+\bar{V}^{3 / 4}\right)^{2}
$$

According to classical theory, this is, therefore, the minimum value of $\alpha$ for which reflecting solution exists. The subscript $\mathrm{BF}$ on $\alpha_{\mathrm{BF}}$ indicates that the steady state bifurcates into two different types at $\alpha_{\mathrm{BF}}$ : the steady state corresponds to unidirectional flow of injected current $\alpha$ if $\alpha \leq \alpha_{\mathrm{BF}}$ whereas it starts reflecting if $\alpha>\alpha_{\mathrm{BF}}$. On comparing (34) with (21) it follows that $\alpha_{\mathrm{DR}}=\alpha_{\mathrm{SCL}}$ i.e. the bifurcation of the steady state from unidirectional flow to partially reflecting flow takes place at the same value of current above which there are two solutions for the unidirectional flow, one stable and another unstable. The electric field at the entrance of the diode for $\alpha=\alpha_{\mathrm{BF}}$, obtained using (30), reads 


$$
\bar{E}_{\mathrm{OBF}} \equiv-\left.\left(\frac{\mathrm{d} \bar{\phi}}{\mathrm{d} \bar{z}}\right)\right|_{\bar{z}=0}=\frac{4}{3}\left(1+\bar{V}^{3 / 4}\right) .
$$

This is the same as the one in (22) obtained using the steady state solution for $\alpha=\alpha_{\mathrm{DR}}$.

It may be verified that the current $J_{0}$ corresponding to $\alpha_{\mathrm{BF}}$ is given by (3). Since the bifurcation point marks the onset of reflections, which is one of the characteristic properties of the Child-Langmuir current, the current $\alpha_{\mathrm{BF}}$ at the bifurcation point is identified in Ref. [6] as the Child-Langmuir current. However, the numerical solution of equations shows that reflection does not start at the bifurcation point. It starts only when the injected current exceeds its space charge limiting value $\alpha_{\mathrm{SCL}}$ which is higher than its value at the bifurcation point except when $v_{0}=0$ in which case, a comparison of (2) and (3) shows that

$$
J_{\mathrm{BF}}=J_{\mathrm{SCL}}, \quad v_{0}=0 .
$$

On comparing with (1) it follows that these currents are the same as the Child-Langmuir current $J_{\mathrm{CL}}$.

The behaviour of $\bar{E}_{0}$ for partially reflecting solution is exhibited in Fig. 1 by the branch of the curve above the bifurcation point $B$. That branch is obtained by computing first the fraction $f$ of the transmitted current by solving 33 for a given $\alpha$. The value of $f$ so obtained is substituted in 30 to evaluate $\bar{E}_{0}$.

Before proceeding further, we digress to rewrite Eq.(32) to express the transmitted current $f \alpha$ in the form

$$
f \alpha=\frac{(1+e V / \varepsilon)^{3 / 2}}{\left(1-\bar{z}_{m}\right)^{2}} .
$$

This resembles the expression derived by Langmuir [8] for the thermionic current from a hot cathode emitting into a diode. Langmuir's expression in question reduces exactly to (37) if the cathode temperature is zero whereas it gives the current approximately if the temperature is non-zero but low. It should, however, be emphasised that in the model under discussion, all the electrons are injected in to the diode with the same velocity whereas, in the paper of Langmuir, the electronic velocities at the entrance are distributed according to the Boltzmann distribution. The position $\bar{z}_{m}$ of the potential minimum in (37) determining $f \alpha$ itself depends on $f$. Hence, evaluation of (37) requires a relation between $\bar{z}_{m}$ and $f$ which, in our case, is contained in (31).

The Eq.(33) leads to the following useful expression for the transmitted current,

$$
\alpha_{\mathrm{TR}} \equiv f \alpha=\left[\bar{V}^{3 / 4}+\sqrt{\frac{f}{2-f}}\right]^{2},
$$

for all $\alpha \geq \alpha_{\mathrm{BF}}$. In terms of the unnormalized variables, this reads

$$
J_{\mathrm{TR}}=J_{\mathrm{CL}}\left[\left(\frac{m v_{0}^{2}}{2 e V}\right)^{3 / 4} \sqrt{\frac{f}{2-f}}+\left(1+\frac{m v_{0}^{2}}{2 e V}\right)^{3 / 4}\right]^{2}
$$

for all $J_{0} \geq J_{\mathrm{BF}}$. Since $0 \leq f \leq 1$, this expression, in the limit of vanishing injection velocity $(\varepsilon \rightarrow 0)$ yields

$$
J_{\mathrm{TR}}=J_{\mathrm{CL}}, \quad v_{0}=0, \quad J_{0} \geq J_{\mathrm{BF}},
$$

where $J_{\mathrm{CL}}$ is the Child-Langmuir current (1). This is the current transmitted for any injected current above its space charge limiting value. This value of current is the same as the value of the space charge limiting current (2) corresponding to $v_{0}=0$. It therefore follows that, for $v_{0}=0$, the transmitted current saturates at $J_{\mathrm{CL}}$ as soon as the injected current attains its space charge limiting value.

The saturation current for non-zero injection velocities, obtained from (38) in the limit $f \rightarrow 0$, reads

$$
\alpha_{\mathrm{TR}} \rightarrow \alpha_{\mathrm{GCL}}, \quad \text { as } \quad f \rightarrow 0,
$$

where $\alpha_{\mathrm{GCL}}$ is the saturation current given by

$$
\alpha_{\mathrm{GCL}}=\bar{V}^{3 / 2} .
$$

We call the saturation current in the equation above as the generalized Child-Langmuir current. Its unnormalized form is given by (4).

The finite value of the transmitted current $f \alpha$ in the limit $f \rightarrow 0$ implies that it is achieved when $\alpha \rightarrow \infty$. Thus, the current transmitted across the diode saturates at the value given by (42) when the current injected in it is increased indefinitely.

We assess the validity of the classical theory by comparing its predictions with numerical results in the next section.

\section{NUMERICAL RESULTS}

In this section we present a comparison of the predictions of the steady state theories and numerical computations carried out using the sheet model [7]. The comparison is carried by computing the transmitted current $J_{\mathrm{TR}}$ as a function of the injected current $J_{0}$ ( $\alpha$ is related to $J_{0}$ by Eq. (12)).

The injected current $J_{0}$ in the sheet model corresponds to injecting $N$ sheets per unit time $\left(L /(2 \mathrm{eV} / \mathrm{m})^{1 / 2}\right)$ into the diode, each having a surface charge density $\sigma=J_{0} / N$. By counting the sheets starting from the one closest to the collecting plate, it can be shown that when there are $M$ sheets in the drift space, the electric field on the $k^{\text {th }}$ sheet at the position $z_{k}$ from the entrance plate is given by

$$
E\left(z_{k}\right)=-4 \pi \sigma\left[k-\frac{1}{L} \sum_{j=1}^{M} z_{j}-\frac{1}{2}\right]+\frac{V}{L} .
$$

The (unnormalized) potential at any point $z$ between the $(k)^{t h}$ and $(k+1)^{t h}$ sheet is similarly given by

$\phi(z)=\frac{V z}{L}-\frac{4 \pi J_{0}}{N}\left[z\left(k L-\sum_{i=1}^{M} z_{i}\right)+L \sum_{i=k+1}^{M} z_{i}\right]$ 
where the sheet number is counted from the anode (right) plate. The force equation for the sheet is thus

$$
m \frac{\mathrm{d}^{2} z_{k}}{\mathrm{~d} t^{2}}=e E\left(z_{k}\right)
$$

The numerical procedure consists of solving the coupled set of equations for $z_{i}(t),(i=1,2, \ldots)$ as a function of time by choosing the time-step $\Delta t$ of integration and the rate of injection $N$ of the sheets in such a way that desired accuracy is achieved. Typically, $\Delta t=8.532 \times 10^{-13} \mathrm{~s}$, $N=1000$ for $J_{0}=20000 \mathrm{~A} / \mathrm{m}^{2}$

The numerical computations confirm the theoretical prediction that, for $\alpha \leq \alpha_{\mathrm{SCL}}$, the system approaches a steady state asymptotically as $t \rightarrow \infty$. However, contrary to the premise of the classical theory, the asymptotic behaviour of the system in time is found to be oscillatory if $\alpha>\alpha_{\mathrm{SCL}}$. Hence, for $\alpha>\alpha_{\mathrm{SCL}}$, the predictions of the classical theory are compared with the results of numerical computations averaged over several cycles of oscillations after the initial transients have settled. See Ref. [9] for a plot of oscillating transmitted current as a function of time for injected current in excess of the SCL current in a short-circuited diode.

The Fig.2 is a plot of theoretical predictions and numerically computed values of the transmitted current $J_{\mathrm{TR}}$ as a function of the injected current $J_{0}$ for $\varepsilon=0$ and for $\varepsilon / e V=0.2$ with $L=.008 \mathrm{~m}$ and $V=250 \mathrm{~V}$. The bold straight line represents $J_{\mathrm{TR}}=J_{0}$ which is the prediction of the steady state theory for currents below the space charge limiting value. The square dots on that line are the results of numerical computations indicating agreement between analytical and numerical solutions for currents below the space charge limiting value.

The numerical results of computation for currents exceeding the space charge, carried in the manner outlined above, are represented by the square dots. The dasheddotted straight line (a) is drawn to join the dots corresponding to $\epsilon=0$ whereas the dashed-dotted curve (b) for $\varepsilon / e V=0.2$ joins the dots corresponding to $\varepsilon=0.2$. Recall that the classical theory predicts that if $\varepsilon=0$ then $J_{\mathrm{TR}}=J_{\mathrm{CL}}$ for all $J_{0}$. For the values of the parameters used in the figure, the value of the Child-Langmuir current is $J_{\mathrm{CL}} \simeq 143.9$ which practically coincides with the corresponding numerical results represented by the straight line (a). Hence, the theoretical predictions are in agreement with numerical results for any value of the injected current if $\varepsilon=0$.

The theoretical plot for $\varepsilon / e V=0.2$ is represented by the dashed curve marked "classical theory". It is obtained by solving (33) for $f$ for a given value of $\alpha$ and using the value of $f$ so obtained to evaluate the transmitted current $J_{\mathrm{TR}}=f \alpha$. On comparing the classical theory plot with the corresponding results of numerical computations represented by the curve (b), it is seen that the agreement between the two is not close. For, in disagreement with the monotonic behaviour predicted by the classical theory, the numerical plot exhibits a maxima in $\alpha_{\mathrm{TR}}$ as a function of $\alpha$. The reason behind the appearance of the maxima is left as an open question. However, the asymptotic limit of the numerical plot of
$\alpha_{\mathrm{TR}}$ appears to be in agreement with the prediction (41) of the classical theory. In order to confirm the apparent agreement we examine the asymptotic behaviour of the curve

$$
J_{\mathrm{TR}}=a+b J_{0}^{-\beta}
$$

fitting best the numerical results. The Fig. 3 shows some values of $J_{\mathrm{TR}}$ computed numerically along with the curve (46) fitting best the numerical results as a function of $J_{0}$. The best fit is obtained for $a=193.58, b=2318.83$ and $\beta=0.40$. Hence, (46) approaches asymptotically the value $J_{\mathrm{TR}}=193.58$ which agrees very well with the value $J_{\mathrm{TR}}=189.37$ obtained using the theoretical expression (42). We have observed similar agreement between theoretical prediction and the numerical computations for several other values of the injected velocity.

Finally, we study the behaviour of the (time-averaged) position of the virtual cathode as a function of the injected current $J_{0}$ for $\varepsilon=0.2$. Fig. 4 shows a plot of the time-averaged normalized position of the potential minimum $\left(\bar{z}_{m}\right)$ as a function of $J_{0}$. As in the case of short-circuited diode, $\bar{z}_{m}$ decreases with $J_{0}$. Fig. 4 also shows the prediction of the classical theory (bold line) and the best fit of the function $a_{1}+b_{1} J_{0}^{-\delta}$ (dashed line) for $J_{0}$ in the interval $[1000,20000]$. The value of the parameters are : $a_{1}=-0.007, b_{1}=1.384$ and $\delta=0.335$. Clearly, the position of the virtual cathode moves to the injection plane as $J_{0} \rightarrow \infty$.

\section{CONCLUSIONS}

In this paper, we have sought to generalize the ChildLangmuir law for non-zero injection velocities based on the saturation property of the time-averaged transmitted current. The reason for preferring the saturation property for the said generalization is its importance in numerical simulations. For, as stated in the Introduction, the emission characteristics of cathode and the details of the emission mechanism do not play any role in determining the transmitted current if that current is independent of (or weakly dependent on) the injected current. This is the case when the current emitted from the cathode surface $\left(J_{0}\right)$ is more than what gives rise to the saturation value of the transmitted current. This condition simplifies considerably the task of numerical simulation.

Thus, if we characterize the Child-Langmuir current as the saturation current then $\alpha_{\mathrm{GCL}}$ given in Eq. (42) is the Child-Langmuir current generalized to non-zero velocities of injection. That expression for the saturation current is derived by assuming a steady flow of current under the condition that a part of the current is reflected from the virtual cathode. Eq. (42) is found to be in fairly good agreement with numerical computations. In contrast to the case of zero velocity of injection for which saturation is attained at the space charge limiting value of the injected current, the saturation in the case of nonzero velocities is attained only asymptotically. Hence, though the details of the emission characteristic of cathode may be ignored safely in numerical simulations for 
any injected current above its space charge limiting value in case $v_{0}=0$, the injected current for $v_{0} \neq 0$ needs to be very much above its space charge limiting value for cathode characteristics to become unimportant.

\section{APPENDIX A: STEADY STATE IN LAGRANGIAN FORMALISM}

In this Appendix we present steady state solution of the Eq.(16) describing the flow of current in Lagrangian formalism.

Integration of (16) yields

$$
\frac{\partial v\left(t, t_{0}\right)}{\partial t}=-\frac{e}{m}\left[E_{0}(t)+4 \pi \int_{t_{0}}^{t} \mathrm{~d} \tau J_{0}(\tau)\right] .
$$

This equation is arrived at by noting that the electron at time $t=t_{0}$ is at $z=0$. Hence, the initial condition is the same as the condition at $z=0$ so that, by virtue of the last equation in $(5), \partial v\left(t, t_{0}\right) / \partial t=-e E_{0} / m$ at $t=t_{0}$. The equation (A1) may be integrated further to obtain expressions for $v\left(t, t_{0}\right)$ and $z\left(t, t_{0}\right)$. Like $(\mathrm{A} 1)$, those equations involve two unknown functions: the field and the current at all times at the entrance plate. The current at the entrance plate consists of the current being constantly pumped in and the current returning, if any, after reflection from some position between the plates. Our interest here is in the steady state without returning currents. The current at the entrance plate in that case is the same as that being pumped in i.e.

$$
J_{0}(t)=J_{0}=\rho_{0} v_{0}=-\left|\rho_{0}\right| v_{0} .
$$

We normalize the variables as in (17) and integrate (A1) in the absence of returning currents to get

$$
\begin{aligned}
\bar{v}\left(\bar{t}, \bar{t}_{0}\right)= & 1-\frac{1}{2} \int_{\bar{t}_{0}}^{\bar{t}} \mathrm{~d} \tau \bar{E}_{0}(\tau) \mathrm{d} \tau+\frac{\alpha}{9}\left(\bar{t}-\bar{t}_{0}\right)^{2}, \\
\bar{z}\left(\bar{t}, \bar{t}_{0}\right)= & \left(\bar{t}-\bar{t}_{0}\right)-\frac{1}{2} \int_{\bar{t}_{0}}^{\bar{t}} \mathrm{~d} \tau(\bar{t}-\tau) \bar{E}_{0}(\tau) \\
& +\frac{\alpha}{27}\left(\bar{t}-\bar{t}_{0}\right)^{3} .
\end{aligned}
$$

The unknown function $\bar{E}_{0}(t)$ in these equations is found by imposing the boundary conditions (7). That task is simplified in the steady state because the field $E_{0}(t)$ at the entrance plate is then independent of time reducing (A3) and (A4) to the forms which are functions only of the time difference $t-t_{0}$ :

$$
\begin{aligned}
& \bar{v}\left(\bar{t}, \bar{t}_{0}\right)=1-\frac{1}{2}\left(\bar{t}-\bar{t}_{0}\right) \bar{E}_{0}+\frac{\alpha}{9}\left(\bar{t}-\bar{t}_{0}\right)^{2}, \\
& \bar{z}\left(\bar{t}, \bar{t}_{0}\right)=\left(\bar{t}-\bar{t}_{0}\right)-\frac{1}{4}\left(\bar{t}-t_{0}\right)^{2} \bar{E}_{0}+\frac{\alpha}{27}\left(\bar{t}-\bar{t}_{0}\right)^{3} .
\end{aligned}
$$

Note that these equations already incorporate the boundary condition at $z=0$. The boundary condition (7) at $z=L$, restated in terms of the velocity by invoking the conservation law (10), leads to the expression (18) for velocity at the collector plate in terms of its potential.
Now, if $\bar{T}(1)$ is the time that an electron takes to reach the collector plate after its entry in to the diode then the Equations (A5) and (A6) yield

$$
\begin{aligned}
\bar{v}_{1} & =1-\frac{1}{2} \bar{T}(1) \bar{E}_{0}+\frac{\alpha}{9} \bar{T}^{2}(1), \\
1 & =\bar{T}(1)-\frac{1}{4} \bar{T}^{2}(1) \bar{E}_{0}+\frac{\alpha}{27} \bar{T}^{3}(1) .
\end{aligned}
$$

The two equations above determine the unknowns $\bar{E}_{0}$ and $\bar{T}(1)$. On eliminating $\bar{E}_{0}$ between these equation, the transit time $\bar{T}(1)$ is found to obey the cubic

$$
\bar{T}^{3}(1)-\frac{27}{\alpha}\left(1+\bar{v}_{1}\right) \bar{T}(1)+\frac{54}{\alpha}=0 .
$$

Of course, only the real positive roots of this equation are physically acceptable. The electric field at the entrance plate, obtained by substituting an acceptable solution of (A9) in (A7) is

$$
\bar{E}_{0}=\frac{2}{\bar{T}(1)}\left[\frac{\alpha}{9} \bar{T}^{2}(1)+1-\bar{v}_{1}\right] .
$$

Now, the nature of the roots is determined by the sign of the discriminant

$$
\Delta=\frac{1}{4} q^{2}+\frac{1}{27} p^{3}
$$

where

$$
p=-\frac{27}{\alpha}\left(1+\bar{v}_{1}\right), \quad q=\frac{54}{\alpha}
$$

so that

$$
\Delta=\left(\frac{27}{\alpha}\right)^{2}\left(1-\frac{1}{\alpha}\left(1+\bar{v}_{1}\right)^{3}\right) .
$$

If $\Delta \leq 0$ then the three roots are real whereas one root is real and the other two complex conjugate of each other if $\Delta>0$.

Note that $\Delta \leq 0$ and hence the three roots are real if $\alpha \leq\left(1+\bar{v}_{1}\right)^{3}$. The roots in this case are

$$
\begin{aligned}
& \bar{T}_{1}(1)=-2 A^{1 / 3} \cos \left(\frac{\theta}{3}\right), \\
& \bar{T}_{2}(1)=2 A^{1 / 3} \cos \left(\frac{\theta+\pi}{3}\right) \\
& \bar{T}_{3}(1)=2 A^{1 / 3} \cos \left(\frac{\pi-\theta}{3}\right),
\end{aligned}
$$

where

$$
\begin{aligned}
A & =27\left[\frac{1}{\alpha}\left(1+\bar{v}_{1}\right)\right]^{3 / 2}, \\
\tan (\theta) & =\sqrt{\left(\frac{1}{\alpha}\left(1+\bar{v}_{1}\right)^{3}-1\right)}, \quad 0 \leq \theta \leq \frac{\pi}{2} .
\end{aligned}
$$

The root $\bar{T}_{1}(1)$, being negative, is physically unacceptable. Hence, $\bar{T}_{2}(1)$ and $\bar{T}_{3}(1)$ are the admissible times of 
transit. The expression for the electric field (A10) at the entrance of the cathode due to the positive roots assumes the form

$$
\begin{aligned}
\bar{E}_{0}= & \frac{2 \sqrt{\alpha}}{3\left(1+\sqrt{\bar{v}_{1}}\right)}\left[\cos \left(\frac{\pi \pm \theta}{3}\right)\right]^{-1} \\
& \times\left[4\left(1+\bar{v}_{1}\right) \cos ^{2}\left(\frac{\pi \pm \theta}{3}\right)+1-\bar{v}_{1}\right] .
\end{aligned}
$$

We now examine the question of admissibility of $\bar{T}_{2}(1)$ and $\bar{T}_{3}(1)$ based on other physics considerations. We will see that the slower of the two transit times, $\bar{T}_{3}(1)$, corresponds to negative velocity in some region if $0 \leq \alpha \leq$ $\left(1+\bar{v}_{1}^{3 / 2}\right)^{2}$ and to positive velocity all through the diode but an unstable steady state if $\left(1+\bar{v}_{1}^{3 / 2}\right)^{2}<\alpha \leq\left(1+\bar{v}_{1}\right)^{3}$.

To that end, let $\bar{v}_{m}$ be the minimum velocity at some place in the diode and let $\bar{T}_{m}$ be the time that an electron takes to reach that position so that $\mathrm{d} \bar{v} / \mathrm{d} \bar{T}=0$ at $\bar{T}=$ $\bar{T}_{m}$. On solving (A3) (with $\bar{t}-\bar{t}_{0}=\bar{T}$ ) for $\mathrm{d} \bar{v} / \mathrm{d} \bar{T}=0$, it follows that

$$
\begin{aligned}
& \bar{T}_{m}=\frac{9}{4 \alpha} \bar{E}_{0}, \\
& \bar{v}_{m}=1-\frac{9}{16 \alpha} \bar{E}_{0}^{2} .
\end{aligned}
$$

This equation shows that the minimum velocity is negative if either $\bar{E}_{0}<-4 \sqrt{\alpha} / 3$ or $\bar{E}_{0}>4 \sqrt{\alpha} / 3$. However, (A5) shows that if $\bar{E}_{0} \leq 0$ then $\bar{v} \geq 0$. Hence, the condition for the velocity to become negative in some region in the diode is $\bar{E}_{0}>4 \sqrt{\alpha} / 3$ which, in conjunction with (A10), reads

$$
\left(\frac{\sqrt{\alpha}}{3} \bar{T}(1)-1-\sqrt{\bar{v}_{1}}\right)\left(\frac{\sqrt{\alpha}}{3} \bar{T}(1)-1+\sqrt{\bar{v}_{1}}\right)>0 .
$$

The positivity of $\bar{T}(1)$ implies that (A19) can be satisfied if

$$
\bar{T}(1)>\frac{3}{\sqrt{\alpha}}\left(1+\sqrt{\bar{v}_{1}}\right) .
$$

This condition, for $\bar{T}(1)=\bar{T}_{2}(1), \bar{T}_{3}(1)$ given in (A14)), assumes the form

$$
\cos \left(\frac{\pi \pm \theta}{3}\right)>\frac{1}{2}\left(1+\sqrt{\bar{v}_{1}}\right)\left(1+\bar{v}_{1}\right)^{-1 / 2} .
$$

It is easy to check that this implies $\cos ((\pi \pm \theta) / 3))>$ $1 / 2$ which, in turn, implies $(\pi \pm \theta) / 3<\pi / 3$. Since $\theta$ is positive, the last inequality can be satisfied only by $\pi-\theta$. Thus, the minimum velocity can become negative only for $\bar{T}_{3}(1)$. Hence, $\bar{T}_{2}(1)$ is the only acceptable root for those values of $\alpha$ for which $\bar{T}_{3}(1)$ leads to negative velocities. Those values of $\alpha$ may be identified by noting that the condition (A21) for $\bar{T}_{3}(1)$ implies

$$
\pi-\theta<3 \cos ^{-1}\left[\frac{1}{2}\left(1+\sqrt{\bar{v}_{1}}\right)\left(1+\bar{v}_{1}\right)^{-1 / 2}\right] .
$$

Using the trigonometric relation $\cos (3 x)=4 \cos ^{3}(x)-$ $3 \cos (x)$, and the defining relation (A15) of $\theta$, it is straightforward to show that (A22) holds if

$$
\alpha \leq\left(1+\bar{v}_{1}^{3 / 2}\right)^{2} \equiv \alpha_{\mathrm{DR}}
$$

where $\alpha_{\mathrm{DR}}$ is given by (21). In other words, if $\alpha$ satisfies the condition (A23) then the root $\bar{T}_{3}(1)$ satisfies the condition (A20) required for the velocity to become negative in some region in the diode leaving $\bar{T}_{2}(1)$ as the only acceptable root in the range $0 \leq \alpha \leq \alpha_{\mathrm{DR}}$ whereas both, $\bar{T}_{2}(1)$ and $\bar{T}_{3}(1)$, are acceptable if $\alpha_{\mathrm{DR}} \leq \alpha<\left(1+\bar{v}_{1}\right)^{3}$. The $\alpha_{\mathrm{DR}}$ denotes the value of the current above which there are two roots. However, the linear stability analysis shows that the root $\bar{T}_{3}(1)$ is unstable. Hence, only $\bar{T}_{2}(1)$ corresponds to a stable steady state in the range $0 \leq \alpha \leq\left(1+\bar{v}_{1}\right)^{3}$.

The discriminant $\Delta>0$ if $\alpha>\left(1+\bar{v}_{1}\right)^{3}$. The cubic (A9) determining the transit time in this case has one real and a pair of complex conjugate roots. Since the transit time is a real quantity, the real root is the only one which needs to be examined further for its acceptability. To that end, we note that the product of the roots of (A9), given by $-54 / \alpha$, is negative. Hence, the real root in case of $\Delta>0$ must be negative. Since the time of transit is a positive number, the negative root is physically unacceptable. The cubic (A9), therefore, does not admit physically acceptable solution i.e. the system does not approach a time-independent steady state having unidirectional flow of current if $\alpha>\alpha_{\mathrm{SCL}}$ where

$$
\alpha_{\mathrm{SCL}}=\left(1+\bar{v}_{1}\right)^{3}
$$

is the space charge limiting current: The space charge limiting current is the value of the current above which the flow of current is no longer unidirectional and timeindependent. It is also the maximum current that can be transmitted across the diode even when the injected current is increased indefinitely.

The solution of (A9) for $\alpha=\alpha_{\mathrm{SCL}}$ gives the transit time as

$$
T_{\mathrm{SCL}}(1)=\frac{3}{1+\sqrt{\bar{V}}}
$$

which, on substitution in (A10) yields

$$
\bar{E}_{\mathrm{SCL} 0}=\frac{4}{3}(1+\sqrt{\bar{V}})
$$

This is the electric field at the entrance of the diode when the value of the injected current $\alpha=\alpha_{\mathrm{SCL}}$.

[1] A.Ya.Ender, H.Kolinsky, V.I.Kuznetsov, and H.Schamel, Phys.Rep. 328, 1 (2000).

[2] J. W. Luginsland, Y. Y. Lau and R. M. Gilgenbach, Phys. Rev. Lett. 77, 4668 (1996); J. W. Luginsland, Y. Y. Lau, R. J. Umstattd and J. J. Watrous, Phys. Plasmas 9, 2371 (2002). J. J. Watrous, J. W. Luginsland, M. H. Frese, Phys. Plasmas 8, 4202 (2001).

[3] Y. Y. Lau, Phys. Rev. Lett. 87, 278301 (2001); 
[4] K. G. Kostov and J. J. Barroso, Phys. Plasmas 9, 1039 (2002).

[5] P.V.Akimov, H.Schamel, H.Kolinsky, A.Ya.Ender, and V.I.Kuznetsov, Phys.Plasmas 8, 3788 (2001).

[6] S.Liu and R.A.Dougal, J. Appl. Phys. 78, 5919 (1995).

[7] C.K.Birdsall and W.B.Bridges, Electron Dynamics of Diode Regions (Academic Press, New York, 1966).

[8] I.Langmuir, Phys.Rev. 21, 419 (1923).

[9] A.E.Dubinov and I.A.Elfimova, Appl. Phys. Lett. 81, 1530 (2002)

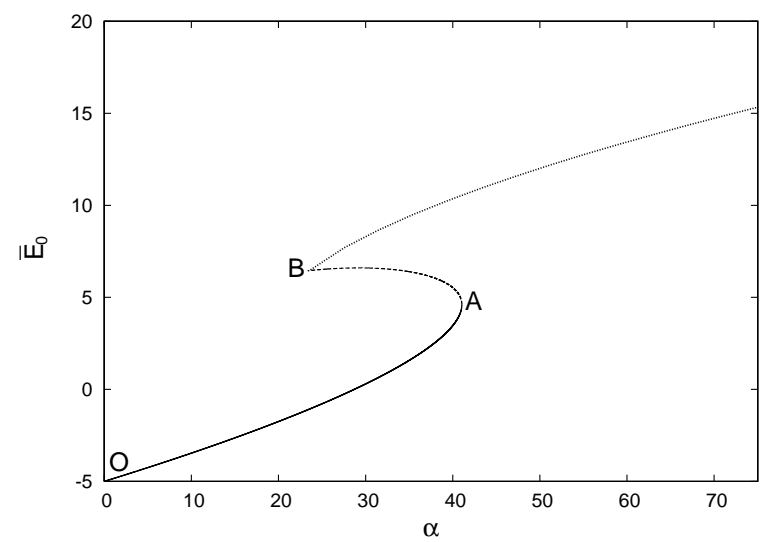

FIG. 1. The electric field at the cathode $\bar{E}_{0}$ plotted as a function of the current $\alpha$ for $\varepsilon / e V=0.2$. The branches OA (stable) and $\mathrm{AB}$ (unstable) correspond respectively to $\bar{T}_{2}(1)$ and $\bar{T}_{3}(1)$ given by (A14). The uppermost branch of the curve for $\alpha>\alpha_{\mathrm{DR}}\left(=\alpha_{\mathrm{BF}}\right)$ (point B) is a result of the classical theory.

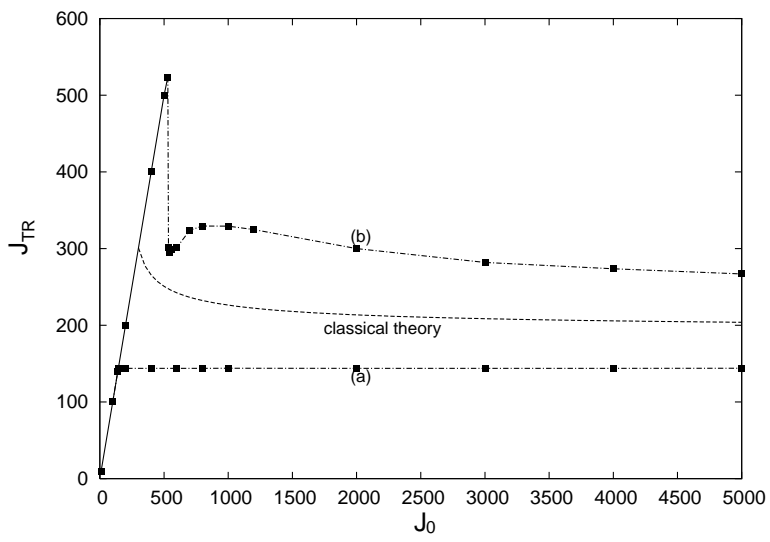

FIG. 2. The transmitted current density, $J_{\mathrm{TR}}$, plotted against the injected current density, $J_{0}$, for two different value of $\varepsilon / \mathrm{eV}$ with $L=0.008 \mathrm{~m} V=250 \mathrm{~V}$. The current density is in units of Amp $/ \mathrm{m}^{2}$. The square dots are the results of the numerical computation. The bold line is $J_{\mathrm{TR}}=J_{0}$. The straight line (a) is drawn to join the results of numerical computations for $\varepsilon=0$ while the curve (b) joins the numerical results for $\varepsilon / e V=0.2$. The dotted line marked "classical theory" is the prediction of classical theory for $\varepsilon / e V=0.2$.

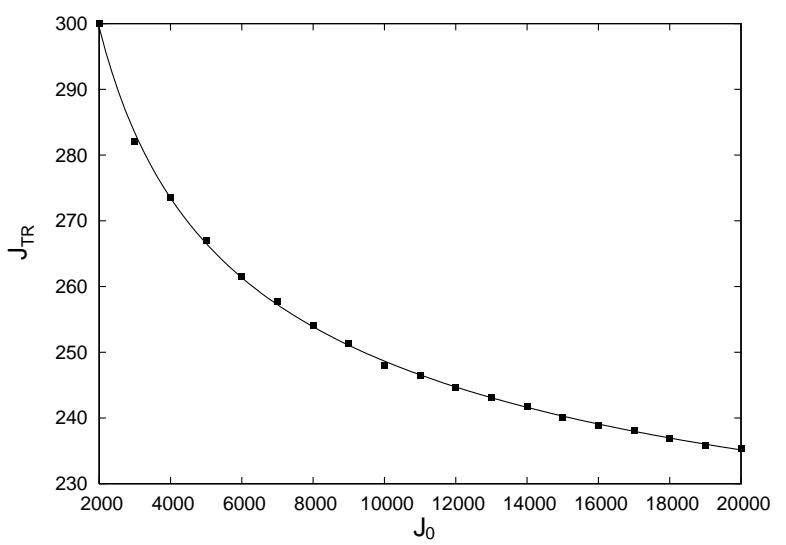

FIG. 3. A fit of the transmitted current density using $J_{\mathrm{TR}}=a+b J_{0}^{-\beta}$ as a function of the injected current density $J_{0}$. The current density is units of Amp $/ \mathrm{m}^{2}$. The solid line is the best fit while the squares mark the numerically computed values of the transmitted current using the sheet model.

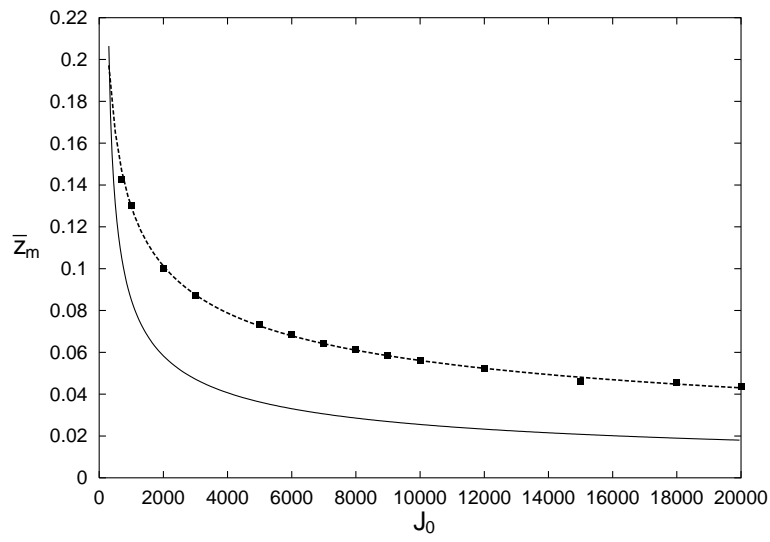

FIG. 4. A plot of the time-averaged normalized position of the virtual cathode (solid squares) as a function of $J_{0}$. Also shown are the prediction of the classical theory (bold line) and the best fit using the function $a_{1}+b_{1} J_{0}^{-\delta}$ (dashed line). 